\title{
Competencies of BSc and MSc programmes in Electrical engineering and student portfolios
}

\author{
Ton J.Mouthaan, R.W. Brink, H.Vos \\ University of Twente, fac. of EE, The Netherlands \\ Corr. author a.j.mouthaan@el.utwente.nl
}

\begin{abstract}
General goals of a BSc and MSc in Electrical Engineering are formulated, leading to a 'mission' statement clarifying the rationale behind the programmes in relation to the needs of society. These goals are made operational in terms of competencies that engineers educated through our programmes are required to have in order to operate successfully in society. These competencies are detailed to a level that they can correspond to individual items in the curricula. A methodology is described for the evaluation of the level at which the students have acquired the needed competencies. It involves a portfolio of self evaluation that is kept by the students and records kept by staff, together constituting an individual portfolio describing the students abilities that can be used as part of a CV.
\end{abstract}

\section{General goals of BSc and MSc programmes in Electrical Engineering}

There is a large and even increasing demand for Electrical Engineers trained at university level in society. The average time of unemployment after graduation is very short graduates work generally in areas for which their university education in EE is indispensable. There is a need to clarify in a practical way what 'university education in EE' means. There are several reasons for that:

- The new diploma structure forces Europe to define end-terms that distinguish BSc from MSc and from BEng (professional Bachelor for which the title Bachelor of Engineering is used here); the distinction must be 'tangible' i.e. verifiable in the execution of professional activities.
- For the process of accreditation of our curricula it is imperative to state and verify our mission.

- We should be able to translate this mission into a study programme that trains students in the competencies that are required to perform as a successful engineer.

- It should lead to a scheme that the students and staff can use for assessment.

The most general professional role of university educated Electrical Engineers is that they perform innovative 'design'. Designing is used in a broad way: the creation of a ('electrical') system with some required functionality. This system can be a small unit that performs according to detailed technical specifications or a complete end product that fulfills a need of society. The adjective 'innovative' is added to design to emphasise the fact that it is expected from university educated engineers that they develop new concepts/methodologies/components/principles. The emphasis on 'design' rather than 'research' and/or 'development' is essential; all activities of Electrical Engineers are performed in the context of an application. A clear perception of this application (societal, -multidisciplinary- engineering, financial and manufacturability dimensions) requires a creativity that finds its roots in a focus on human interest. The 'design' activity encompasses all: a methodology, ability to analyse, recognise/define/perform research activities and an ability to perform these tasks in a business environment. Design at university level requires research to develop new concepts so research is essential for all university educated engineers. Design at BSc level is more 'basic', less independent perhaps less innovative than at MSc level, at least not as specialised as MSc. BSc must differ from BEng. in level of abstraction needed (BEng more mechanistic). 


\section{Description of a Bachelor in Electrical Engineering}

- A Bachelor is an academically educated person, prepared to work under supervision of an academic (Masterlevel) engineer in innovative design (and research and development activities as part thereof).

- The design activities pertain to 'low current', i.e. general signal processing systems in optical, electrical, electro-mechanical or electro-magnetic domain, involving hard and/or software.

- A university trained Bachelor has an attitude of

- curiosity, broad interest, initiative, independence,

- reflection about the broader context of engineering problems and his own position towards this context, i.e. being critical,

- learning new subjects, analysing in an abstract way,

- interest in distinguishing between essential and ad hoc ideas, justification of generalisations, and to be creative in synthesising results.

- An Electrical Engineering Bachelor has such knowledge and understanding of the theory and methodology in the field of Electrical Engineering (except for power engineering) that he is able to solve supervised but independently, Electrical Engineering problems. He should be able to acquire new knowledge when needed and integrate this rapidly. In general, the problems he is faced with, are problems that are not solvable with only standard techniques.

- He must be able to cope with a general, rather vague description of his task, to analyse the problem by formulating specified goals, structure the problem in main and secondary aspects, to model these aspects, and structure the road towards a timely solution.

- He has experience in participating in a multidisciplinary group, being aware of group processes and his own role including his own strong and weak points and he can act accordingly. He should be able to communicate effectively and structured with his colleagues and his supervisor.

The main differences with a Master profile are:

- the specialised knowledge a Master has acquired and is able to apply,

- the complexity of the projects he can tackle,

- the ability to do independent research,

- the ability to be innovative in system design.

A Masters programme therefore will provide this specialised knowledge and train extensively in research to achieve innovative designs. Engineers at Master level will carry responsibility for project execution and definition. The programme has to focus on (industrial) application and build on the competencies related to implementation of design and reflection on societal impact of innovations. Because of the more limited responsibilities of Bachelors, it is required that Bachelors have a basic awareness of the limited scope of EE-methodology and accept and work with differences in paradigms of various scientific areas.

The kind of problems a Bachelor is able to solve, is more limited in scope and complexity; in general, a Bachelor is not contributing directly to innovations, but of course his work may be part of an innovating project. However, a Bachelor is a potential Master. Because of the limited training in the Bachelor programme for innovation -which is the main asset a university education can offer because of its relation between research and education- it should not be standard practice for university trained Bachelors to leave their training for employment. Their training has brought them to an entry level for Masters where they can be made to render a large added value to society as the prime motors for technological development. Individual students can always opt for a career in society and perhaps take up further education later. The BSc degree certainly has market value, but a Masters programme can increase that value tremendously.

The main difference with a professional Bachelor, BEng is the much stronger profiling of the latter towards a career on the employment market. Visible differences in profile are in the general academic attitude, the omission of a general and thorough basic theoretical background, in the ability to see relations and recognise concepts, the ability to cope with rather vaguely formulated problems, the ability to structure problems and the way to solutions. A MSc will also be able to acquire and integrate new knowledge more rapidly. A BSc is less able to design at product level than a BEng but operates more on conceptual level. For students with a BEng degree admission to a MSc programme will require filling in this gap in academic attitude. 


\section{Description of a Master in Electrical Engineering:}

- A Master has the main competencies of a BSc. In case students are admitted with essential omissions, these should be repaired in the first phase of the Masters programme.

- A Master has specialised advanced knowledge in at least one of the areas of Electrical Engineering: Microsystems and Microelectronics, Mechatronics, Telematic Networks, Embedded Systems or Biomedical Technology.

- A Master has experience in working in industry-related projects and has acquired the ability to be effective in a multidisciplinary environment.

- A Master is able to work at the frontier of design (and the research and development that is needed), and is innovative, contributing to breaking the frontiers of current technology or understanding. He defines his own design/research goals within the limits of his job, judges which parts of the problem need further analysis, carries out these analyses on abstract level, proposes experiments and carries them out in a methodologically correct way.

- A Master is able to understand, on a general level, areas adjacent to his own area of specialisation and use this understanding in the context of his own work. He is able to appreciate new knowledge of other disciplines (if necessary also of non-technical areas) and integrate this in his work.

- A Master carries responsibility as a leading member a of multidisciplinary design (or research/development) group and needs a broad scope, e.g. with respect to the economical aspects of his work, or the impact of technological innovation on society. He needs to be a serious partner in discussions on aspects regarding the setting and societal environment of his work.

\section{List of competencies and relation to curriculum items}

There is no unique definition of competency and how it relates to knowledge. Here we refer to knowledge as a more passive state of mind and a mechanical application in standard situations (like knowledge obtained in an isolated course). Knowledge also encompasses the capability to use methods or select methods in solving specific problems in standard situations (like convolutions, transformations, solving differential equations). Knowledge carries the potential to become a competency if used in a collective way and effectively to reach a goal. A Competency hence makes use of a complex of knowledge and focuses on the use of knowledge in non-standard situations. A certain hierarchy is present between knowledge and competence and within the competence level itself; the ultimate competency is that of an engineer, but at this level there is no possibility of ordering so competencies are described here on a level that allows identification as trainable entity. Ultimately also competencies have only meaning if they appear present as a complex.

Necessary competencies can only be derived from an observation of professional practice. This practice is very diverse, yet the mission statement that has been derived from the profile of both Bachelor and Master level engineers is considered to be generic for all professional situations. Not all students will be employed such that all aspects of the profile are present in their jobs, but we perceive it our role to prepare them with the given profile.

A useful division that can be made is one in competencies that are oriented on the discipline of Electrical Engineering and competencies that relate to personal effectiveness. These professional and personal competencies are both needed to become a successful engineer. To be able to present this taxonomy of competencies in a graph both areas can be further divided more related to types of scientific or personal activity (analysis, methodology, individual/team-related competencies, and general attitudes like life-long-learning and societal attitude). See the picture below.

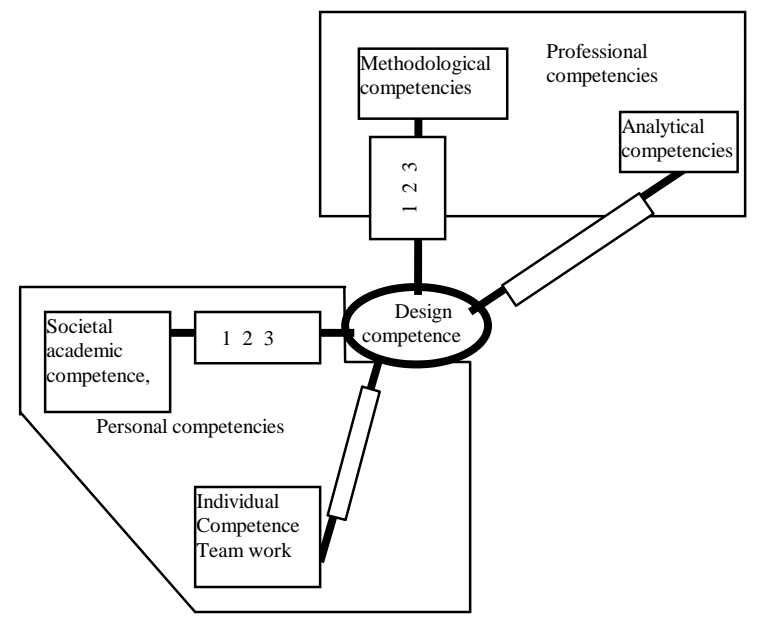

Figure 1: Taxonomy of competencies 
For EE, the professional competencies pertain to methodology and analysis and research tools. These are somewhat independent competencies; the first one relates to the ability to structure (systems level, recognising/describing/specifying sub systems), analysis/research competencies relate to the collected toolbox of knowledge and procedures (modelling, maths and physics to describe and calculate).

Personal competencies make it possible to use professional competencies effectively (communication skills, ability to work in teams), but also relate to attitude (creativity, curiosity, and ability to acquire new knowledge, selfassessment and ability to self-improvement). Personal competencies relate also to view on society, responsibility, ethics, wider scope.

It is obvious that most of the time in the curriculum will be spent on the professional competencies. However a significant conclusion from feedback from the field is that personal competencies matter equally important in later employment. Hitherto personal competencies are developed explicitly only in projects, mostly combined with professional competencies. The number of (group) projects in the curriculum has increased considerably during the last 8 years for both didactical (motivation for study of theory) reasons and for the development of soft skills.

A distinction between competencies at BSc level and MSc is needed. Referring to the mission statement, a Master will be competent (have acquired a complex of knowledge and can use that) in a specialised field. A more fundamental difference will be in the competency to carry out independent research and define/recognise research questions. This means that competencies must be stated in some qualitative way. A common method is to use e.g. a 5-point scale to indicate the level of competence. (Refer to part 3 on evaluation.)

The competencies along each axis are now expanded. Competencies more related to MSc are in Italics.

\section{Methodology}

1. specify, propose and defend design proposals

2. generalise design questions, describe hierarchy, summarise design process in abstract terms

3. translate a design question into a design flow
4. making and evaluating choices made in the design flow

5. translate general design ideas to concrete design specifications (given demands set by practical circumstances)

6. specifying design specifications for (sub)systems

7. recognise and formulate research questions/experiments

8. explore new avenues for problem solving, integrate new techniques in design flow

9. judge and define research activities, translate research products for use in design context

10. execute a design cycle, effective and efficient synthesis of partial results

11. use appropriate modelling and simulation tools to describe behaviour of system

12. realise and test (part of) hard- and/or software

13. compare modelling and realisation results, derive conclusions and terms for possible new iteration

14. independent judgement of design processes

\section{Analysis}

1. find and comprehend literature, judge products of research

2. integrate tools and techniques, ability to abstract, recognise universal concepts

3. make a research plan

4. carry out a given research plan

5. formulate hypothesis and test validity

6. use a base of techniques and tools

7. judge how and when to use specific techniques

8. comprehend new techniques

9. effective and efficient modelling (right level of abstraction), use appropriate modelling languages

10. contribute to literature

\section{Team work and individual Attitude}

1. structuring group work

2. attitude of curiosity

3. creativity

4. comprehend general literature on EE

5. understanding and controlling group dynamics

6. use reflection techniques to facilitate group processes

7. enter into process of independent learning and mastering 
8. comprehend specialised literature

9. explore resources for information and effective and efficiently absorb the information

10. attitude of self assessment

11. ability of self adjustment

12. verbal communication (self confidence, adjust level of detail/abstraction to audience, presentation techniques)

13. written communication (in group, for general public, for customer/boss)

14. independence in judgement, convincing in argumentation

15. recognise shortcomings in knowledge

\section{Societal and academic}

1. give meaningful contribution to societal impact of developments in EE

2. enter into meaningful discussion on general developments in adjacent disciplines

3. affinity to follow developments in bordering disciplines

4. ability to reflect on historical perspective of EE

5. use general products of research and developments from bordering disciplines

6. reflect with sense of responsibility on personal contribution to EE

7. defend ethics of personal choices related to work field

8. overview on generalisms in methodology in general science

As competencies refer to complexes of knowledge they can best be tested in the framework of design projects. This means that it must be clear what different projects aim for. It is imperative that the design projects in the Bachelor and Master programmes follow a hierarchy.

\section{Design, learning to design and testing competencies}

The ability to do innovative design is the ultimate competency trained in our programmes. In all projects in our curriculum this competency and the partial competencies needed are built up in a hierarchical way and integrated.

Design is interpreted as a complex skill or competence. Herein partial skills can be distinguished that are necessarily practised in a hierarchical way. Each consecutive activity of the students trains a number of competencies as listed in the taxonomy and the progression for students follows a 'backwards' path: their first experiments deal with (nearly) completed systems and gradually they learn to design starting from a less detailed level.

To enhance the focus of evaluation to competencies rather than knowledge a digital portfolio is developed, using the taxonomy of competencies and a scaling from 1-5 for each competence.

This portfolio is 'filled' when evaluating the projects in the curriculum by staff reviews (and/or through student assessment) of project work. After graduation, the taxonomy can be the core of the CV of the graduate. In digital form, in a personal part of the portfolio filled by the student only, the student can make a link to project documents or other results and thus create a CV that could be used for his further career. In a digital form this portfolio can be implemented as a web site, where specific parts are open for writing (/reading) for students/staff. This individual portfolio can also be used for submission of products showing development of specific competencies.

Formal examination will be based on the grades of courses and projects. Before using the scored taxonomy as final evaluation experience has to gained in the validity and appropriateness of the system and the best way to implement it. 

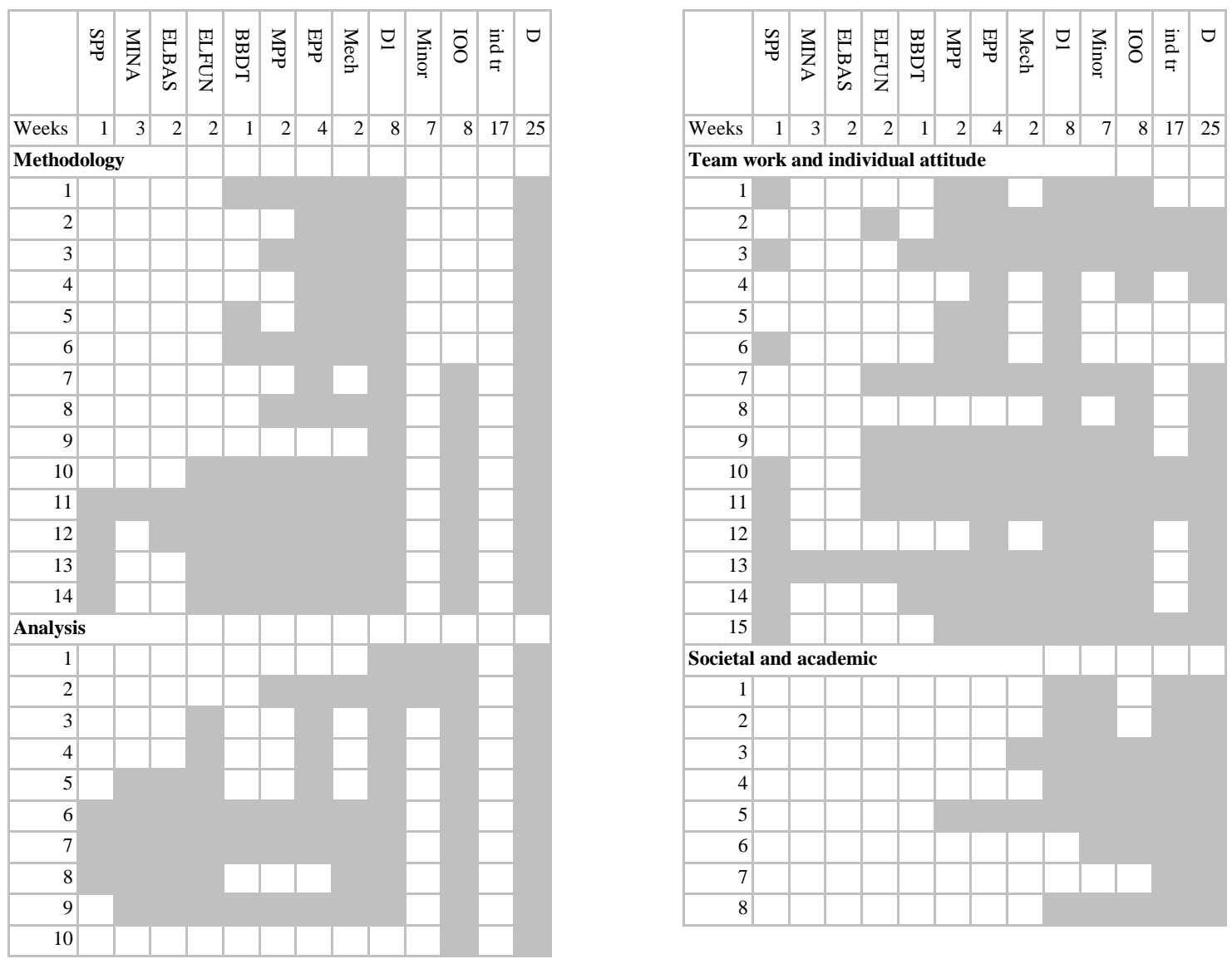

Figure2:

Built up of comptencies through projects 